http://jmscr.igmpublication.org/home/

ISSN (e)-2347-176x ISSN (p) 2455-0450

crossref DOI: https://dx.doi.org/10.18535/jmscr/v9i11.33

Journal Of Medical Science And Clinical Research

\title{
A Study on Current Status of Syndesmotic Injuries
}

\author{
Author
}

\section{Dr Rahmat Ali (Ms (Ortho), MCh (Ortho) )}

Professor and H.O.D (Ortho department), Maharishi Vashishtha Autonomous State Medical College, Basti, UP, India

\section{Abstract}

Syndesmotic injuries are common after ankle fractures. This review aims to present current concepts about the diagnosis and treatment of these injuries.

Keywords: Ankle fractures, Ankle joint, Malleolar fracture, Syndesmosis, Syndesmotic injury.

\section{Introduction}

Our understanding of the ankle complex, syndesmosis injuries, and significance of syndesmotic instability has evolved in the last 50 years shifting from no treatment at all to fixing all the fractures. In recent years, it has been recognized that not all syndesmotic injuries require stabilization. Recently, a survey of the Orthopaedic Trauma Association (OTA) members showed a high grade of variability and controversy in the treatment of ankle fractures.

\section{Review}

Syndesmotic injuries usually occur after ankle pronation external rotation injuries and can be associated with malleolar fractures. A better understanding of the ankle and syndesmotic anatomy has allowed the orthopaedic surgeon to treat syndesmotic injuries. The medial, lateral, and posterior malleoli are important for ankle stability.

\section{Diagnosis}

Physical exam and clinical signs have not shown to be reliable in diagnosing syndesmotic injuries.
Well-known X-ray parameters that suggest a syndesmotic injury are tibiofibular (TF) overlap ( $>6 \mathrm{~mm}$ in the AP view and $>1 \mathrm{~mm}$ from the tibial plafond in the mortise view), TF clear space $(<6$ $\mathrm{mm}, 1 \mathrm{~cm}$ above the tibial plafond in the AP and mortise views), and medialclear space. It is also suggested using the anterior tibiofibular ratio (ATFR) measured on the lateral view as an additional measure that can aid in the diagnosis of a syndesmotic lesion. Dynamic stability of the ankle should be assessed using either external rotation stress, gravity stress, or weight-bearing (WB) radiographs. It is important to bear in mind that each of these methods has pros and cons, for example, manual stress and WB radiographs are dependent on patient's pain tolerance, which can affect its technique. Obtaining ankle stress radiographs in dorsiflexion and external rotation has shown to predict deltoid ligament disruption. It has been reported to have nearly perfect sensitivity and specificity for deltoid ligament disruption. Weight-bearing radiographs allow evaluation of stability and congruence of the ankle joint while reducing the need for surgery. Weber 
et al. evaluated the use of WB radiographs taken 3-10 days post-injury founding that this approach allows for a pain-free evaluation while being reliable in distinguishing those injuries that require surgery without affecting functional results. In cases in which a syndesmotic lesion is suspected but no definitive diagnosis can be made based on X-rays and clinical history alone, a magnetic resonance imaging (MRI) can be warranted. Evaluate the incisura fibularis, and when fractures are present, the articular extension, impaction, and presence of a posterior fragment. In the setting of an ankle fracture, diagnosis of syndesmotic instability can be made intraoperatively under fluoroscopic guidance using either the hook test or external rotation stress test.

\section{Treatment}

We selected 10 cases of ankle fracture or syndesmotic injury is deemed to require surgical fixation, we must consider the different options to obtain and maintain reduction.

\section{Obtaining of Adequate Reduction}

It is critical to obtain an adequate reduction of the syndesmosis, this can either be performed via indirect or direct methods. It is important to consider the anatomic variabilities of the incisura fibularis. A reduction clamp is the simplest method that can be used to reduce the syndesmosis, but adequate positioning is crucial, as a too anteriorly positioned tine can malreduce the syndesmosis translating the fibula and narrowing the incisura anteriorly and vice versa which results in malreduction. Efforts have been made to identify the most accurate clamp position. Clamp placement along the axis of the ankle joint (i.e., tines should be positioned lateral to the malleolar ridge at the fibula and in the central part of the medial cortex in the tibia) has historically been used. Cosgrove et al. described medial tine placement in the anterior third of the tibial line. The use of the TSA has proven to help guide adequate clamp positioning. Therefore, Sagi et al. recommended a "direct, open visualization of the syndesmosis during reduction maneuver". Recently, Tornetta et al. proposed using the articular surface as a visual landmark during open reduction aligning it with the anteromedial fibular articular surface, by doing so, alignment is further improved when compared to relying on the incisura alone. Bringing back the "ring concept", it is important to evaluate if a posterior malleolus fracture is present. The posterior malleolus is the attachment site for the posterior inferior tibiofibular ligament (PITFL). Thus, disruption of the posterior complex can lead to syndesmotic instability. Even after fixation of this fragment has been achieved, it is important to rule out persistent syndesmotic instability.

\section{Method of Fixation}

Once reduction has been obtained, we faces the question of whether to use a static (screw) or dynamic fixation. Several biomechanical and clinical studies have addressed many of the questions and there seems to be no difference in screw size (i.e., 3.5 - 4.5-mm screws), although resistance to shear stress is better tolerated with $4.5 \mathrm{~mm}$ screws, material (stainless steel or titanium), the use of one or two screws, three or four for cortical, and location (trans- or suprasyndesmotic). Advocates of suture-button devices argue that, since the syndesmosis is a mobile joint, a dynamic stabilization should be biomechanically more favourable as it allows movement while preserving reduction. In their study, there were no statistically significant differences in functional outcomes, malreduction (although higher rates were found with screw fixation), and postoperative complications.

\section{Assessment of Reduction}

Irrespective of the method used, it is important to achieve an adequate reduction of the syndesmosis as it affects fixation stability, ankle biomechanics, functional outcomes, and increases the risk of posttraumatic arthritis secondary to an increase in joint reactive forces. Malreduction has been 
identified in upto $50 \%$ of the cases and "small" differences (2-mm) in the anterior TR distance when compared to the uninjured side showed to correlate with poorer outcomes. Persistent diastasis is often overlooked in plain radiographs; therefore, we must be aware of the methods to evaluate diastasis intraoperatively and postoperatively. To evaluate instability intraoperatively, a hook test can be performed and can be complemented with an external rotation stress examination with fluoroscopic imaging. Stress test can be complemented with a mortise and talar dome. Three-dimensional (3D) imaging has shown promising results and its use has been proposed as an alternative to identify subtle malreductions that are not identified in conventional fluoroscopy and/or postoperative radiographs.

\section{Time of Implant Removal}

There seems to be an agreement regarding implant removal. Literature has shown that there is no additional benefit to screw removal as there is no difference in functional, clinical, or radiographic outcomes. It is important to have an early conversation with patients and explain that screw breakage is a possibility, more importantly, it is crucial to emphasize that this does not affect recovery nor outcomes, and hardware removal is not advisable. There are circumstances in which implant removal should be considered (e.g., persistent pain or syndesmosis malreduction). Early removal (i.e., 8-10 weeks) has been associated with syndesmotic diastasis. It is advisable to wait at least 12 weeks for adequate healing.

\section{Summary}

Despite controversy and lack of consensus in some aspects of the diagnosis and treatment of syndesmotic lesions, irrespective of the reduction and fixation method used, to obtain good results it is essential to achieve an adequate reduction.

\section{Reference}

1. Park SS, Kubiak EN, Egol KA, et al. Stress radiographs after ankle fracture: the effect of ankle position and deltoid ligament status on medial clear space measurements. J Orthop Trauma 2006;20(1):11-18.

DOI:

10.1097/01.bot.0000189591.40267.09.

2. Lambert LA, Falconer L, Mason L. Ankle stability in ankle fracture. J Clin Orthop Trauma 2020;11(3):375-379. DOI: 10.1016/j.jcot.2020.03.010.

3. Bäcker HC, Vosseller JT, Harald B, et al. Weightbearing radiography and MRI findings in ankle fractures. Foot Ankle Spec 2020. DOI: 10.1177/1938640020921571.

4. Großterlinden LG, Hartel M, Yamamura J, et al. Isolated syndesmotic injuries in acute ankle sprains: diagnostic significance of clinical examination and MRI. Knee Surgery, Sport Traumatol Arthrosc 2016; 24(4):1180-1186. DOI: $10.1007 / \mathrm{s} 00167$ 015-3604-x.

5. Warner SJ, Garner MR, Fabricant PD, et al. The diagnostic accuracy of radiographs and magnetic resonance imaging in predicting deltoid ligament ruptures in ankle fractures. HSS J 2019;15(2):115121. DOI: $10.1007 / \mathrm{s} 11420-018-09655-x$.

6. Phisitkul P, Ebinger $\mathrm{T}$, Goetz $\mathrm{J}$, et al. Forceps reduction of the syndesmosis in rotational ankle fractures: a cadaveric study. J Bone Jt Surg - Ser A 2012;94(24): 2256-2261. DOI: 10.2106/JBJS.K.01726.

7. Vetter SY, Beisemann N, Keil H, et al. Comparison of three different reduction methods of the ankle mortise in unstable syndesmotic injuries. Sci Rep 2019;9(1). DOI: 10.1038/s41598-019-51988-y.

8. Sagi HC, Shah AR, Sanders RW. The functional consequence of syndesmotic joint malreduction at a minimum 2-year follow-up. J Orthop Trauma 2012;26(7): 
439-443. DOI: $10.1097 /$ BOT.0b013e31822a526a.

9. Li M, Collier RC, Hill BW, et al. Comparing different surgical techniques for addressing the posterior malleolus in supination external rotation ankle fractures and the need for syndesmotic screw fixation. J Foot Ankle Surg 2017;56(4): 730-734. DOI: 10.1053/j.jfas.2017.01.053.

10. Markolf KL, Jackson SR, McAllister DR. Syndesmosis fixation using dual $3.5 \mathrm{~mm}$ and $4.5 \mathrm{~mm}$ screws with tricortical and quadricortical purchase: a biomechanical study. Foot Ankle Int 2013;34(5):734-739. DOI: 10.1177/1071100713478923.

11. Parker AS, Beason DP, Slowik JS, et al. Biomechanical comparison of 3 syndesmosis repair techniques with suture button implants. Orthop J Sport Med 2018;6(10):2325967118804204 10.1177/2325967118804204.

12. Zhang $\mathrm{P}$, Liang $\mathrm{Y}$, He J, et al. A systematic review of suture-button versus syndesmotic screw in the treatment of distal tibiofibular syndesmosis injury. BMC Musculoskelet Disord 2017;18(1): 286. DOI: 10.1186/s12891-017-1645-7.

13. Rushing CJ, Spinner SM, Armstrong AV, et al. Comparison of different magnitudes of applied syndesmotic clamp force: a cadaveric study. J Foot Ankle Surg 2020;59(3):452-456. DOI: 10.1053/j.jfas.2019. 08.028.

14. Davidovitch RI, Weil Y, Karia R, et al. Intraoperative syndesmotic reduction: three-dimensional versus standard fluoroscopic imaging. J Bone Jt Surg - Ser A 2013;95(20):1838-1843. DOI: 10.2106/ JBJS.L.00382.

15. Walley KC, Hofmann KJ, Velasco BT, et al. Removal of hardware after syndesmotic screw fixation: a systematic literature review. Foot Ankle Spec 2017;10(3):252257. DOI: $10.1177 / 1938640016685153$.
16. Gennis E, Koenig S, Rodericks D, et al. The fate of the fixed syndesmosis over time. Foot Ankle Int 2015;36(10):12021208. DOI: $10.1177 / 1071100715588186$. 